\title{
Arthritis Clinical Trials at a Crossroad
}

With more than a century of combined trial experience, we discuss the demise of pharmaceutical company inflammatory arthritis clinical trials, speaking from our experience in Canada while potentially generalizing to the United States, Western Europe, and other regions where patients have reasonable access to medical care and therapeutics.

The purpose of this editorial is 2-fold: to raise awareness of the critical state of rheumatology clinical trials in Canada; and to describe the increasingly insurmountable barriers facing trialists because of difficulty recruiting owing to trial designs (some requiring substandard care for participation); recent changes in trial operations and management; and the financial burden placed on trialists. The challenges are not unique to rheumatology $y^{1,2}$. Many industry-funded trials are not feasible. This can shift investigators to conducting non-pharmaceutical trials to answer challenging important questions, but the trials are usually extremely underfunded, creating a barrier to participation.

Traditionally, clinical research has been the only means whereby advances in medicine can be translated into excellent patient care. Trial participation facilitates greater acceptance of innovative treatments and the integration of successful treatments into optimal care. There is no replacement for phase II and III clinical trial experience: Clinical trialists are early adopters and comfortable prescribers of new therapies once they are approved because they have acquired invaluable experience of novel products.

In contrast, current trials are mostly bureaucratic and time-consuming to initiate and conduct, and they do not reflect the population studied. In fact, clinical research is becoming increasingly onerous with respect to regulatory and administrative requirements and is very expensive (in people and time resources) while, simultaneously, recruitment has dwindled (years ago, sites could enroll 10 subjects in a study vs 0 to 4 per study today). Thus, incurred costs are not recovered. Moreover, recruitment is limited by trial designs that do not reflect patients within rheumatology practices at most sites, and/or do not reflect optimal standard of care treatment. Timelines to start a trial are lengthy; contract negotiations with sponsors, clinical research organizations (CRO), and local hospitals and universities take inordinate amounts of time; and ongoing study demands on the research staff detract from recruitment. To obtain approval for an open-label extension at some sites involves an entirely new institutional review board submission, new contracts, and new consent forms, with delays so prolonged that the first patients who completed the protocol do not have approval in place to continue in the open-label extension. These issues are not encountered at every site but demonstrate how ridiculous the demands are and how detrimental to subject participation. Part of the problem is that site-specific ethics committees require sponsors to pay another large fee.

Given robust infrastructure and ability to integrate research with patient care, clinical trialists are heavily represented in some notable Canadian disease surveillance registries, biobanking, practice guidelines, and genetics research. This infrastructure has enabled participation in underfunded investigator initiated research that is critical to understanding how to best manage rheumatologic diseases.

Without highly qualified research staff in place and research expertise, there will be a deleterious effect on other arthritis research programs, such as those currently funded by peer-reviewed grants. Investigator-initiated trials in particular will suffer as a result of insufficient alternative funding.

If clinical trials of innovative therapies are not conducted in major countries, it will be difficult to use these treatments appropriately. There are both genetic and contextual differences such as treatment approaches and socioeconomic status that may vary per region. For instance, generalizability to Canadian patients [who are taking combination disease-modifying antirheumatic drug (DMARD), high doses of methotrexate (MTX), have often failed multiple DMARD prior to trial entry, and who have normal inflammatory markers] cannot necessarily be inferred if similar patients are not enrolled in protocols.

Over the last 2 decades, we have seen advances in the clinical management of rheumatoid arthritis, psoriatic arthritis, ankylosing spondylitis, systemic sclerosis, vasculitis, and systemic lupus erythematosus. Yet trial designs and entry criteria required for drug development have not kept pace with medical care and thus no longer reflect the patients seen in typical rheumatology practices in Canada, the United States, and Western Europe.

Members of the Canadian Rheumatology Research

See Delivering future clinical trials in rheumatology, page 18 
Consortium (CRRC) published a peer-reviewed article to encourage changes to protocol designs that better reflect the patient population found in North America and Europe ${ }^{3}$. The recommendations include (1) defining optimal dosing of MTX in trials seeking inadequate responders to MTX; (2) revising criteria for elevated erythrocyte sedimentation rate and/or C-reactive protein to increase eligible patients by 75\% without affecting outcomes; (3) shortening drug washout periods to reduce the time study participants with active disease are without treatment ${ }^{4}$; and (4) obtaining patients' consent before drugs are withdrawn in patients with active disease because this would not be usual standard of care (protocols currently will not enroll patients until certain combinations of drugs are washed out prior to study consent). We must use valid methodology to design protocols that are feasible and generate results that can be translated into best practices in clinical care for our patients.

Recent trends in global drug development (such as initiating protocols before they are fully developed, resulting in several amendments) and the corresponding changes in operations and practices (i.e., use of CRO who lack specific knowledge in rheumatology trials, more legal counsel by all parties resulting in longer and more complex contract negotiations, protocols that do not reflect practice) result in significant challenges. Previously, through years of collaboration, sites and local sponsors worked together to minimize delays and maximize productivity while maintaining research integrity. However, today most trials are outsourced to and managed by global CRO. The current trial delivery model has been cultivated to run standardized and massive global projects, and it is not optimized to ensure success at individual sites or even within a country.

Outsourcing has added more chaos (contractors and subcontractors, each with their own standard operating procedures and templates) and has reduced timely access to critical scientific expertise. Especially, it has exponentially increased inefficient processes, such as lack of expertise in specifics of treatment and protocol knowledge and in answering critical protocol questions, ineffective wording of protocols, institution-specific letters of information and contracts that slow down initiation and approval of research at each site, and budgets that do not reflect the time of extra queries due to the above problems and to tasking monitoring work to sites. Each new requirement put in place to manage global issues (particularly risk management strategies, such as additional training, contract language, etc.) has both staff time and financial implications attached to the implementation at the site level. No doubt some of this reflects the typical "growing pains" associated with global operational expansion, but with the limited view of their own projects, most sponsors and CRO are unaware of the extent of these challenges and the cumulative burden placed on the trial sites.

Growth in institutional administrative requirements has also strained site resources and increased study startup timelines. The downloaded work and trial management chaos is at an all-time high in one of the most cost-constrained and recruitment-challenged environments. It is the intersection of these difficulties that makes the survival of Canadian rheumatology trial sites uncertain; other countries likely face the same difficulties.

A substantial investment of both time and resources is required to develop the expertise and infrastructure necessary to transform a basic medical practice into a sophisticated research site. A recent survey (internal data on file) of 39 CRRC sites in which 22 sites responded found that three-quarters (17/22) said they were unsure about continuing trials beyond 2014. Many have reduced their staff. Several of the most experienced sites in Canada have stopped conducting pharmaceutical trials and these sites are not easily re-established. Younger rheumatologists are reluctant to take on the significant workload, inherent frustration, and corresponding financial risk associated with conducting clinical trials.

Based on our collective expertise, we strongly support the following recommendations (with immediate implementation) to remedy challenges and help to strengthen the national clinical research and trials environment.

\section{Recommendations from the Perspective of the Investigative Site}

1. Develop trial designs that better reflect the patients who will receive the products (reflected in current guidelines ${ }^{5,6}$ ), and good clinical practice (GCP). Regulators play a significant role in shaping industry drug development programs; they must consider and encourage alternative study designs, outcomes, and endpoints through education from regional experts.

2. Exploit every opportunity to shorten the study startup window and increase trial efficiency to maximize the time available for core study activities, including recruitment.

3. Develop a clinical trial agreement template, acceptable to all parties, that could be signed without negotiation, including necessary legal aspects (outlining clear responsibilities/obligations; perhaps country specific). To this end, investigators must be part of agreement template development and be protected from personal legal exposure in study agreements. Recent requirements for site insurance above usual malpractice include covering business losses due to breach of contract, loss of data, etc., and are cost-prohibitive (more than $\$ 5000$ US per site per year), yet do not cover trial-related risks.

4. Change global business practices that are having unintended country-level consequences for patients, trialists, institutions, and sponsors. For example, Canadians will adopt new medications less often if there is no clinical trial experience within the country and opinion leaders have not had early access and experience with novel therapies. We appeal to country pharmaceutical affiliates to preserve

Personal non-commercial use only. The Journal of Rheumatology Copyright @ 2015 . All rights reserved. 
or re-establish some form of local operations relationship with the research sites and to advocate for better practices.

5. Strengthen national leadership where stakeholders can effectively collaborate to inform and drive national efforts to address major operational issues, including facilitating protocol and budget templates and minimizing hurdles from ethics review boards by agreeing to common wording and expedited reviews for certain studies. From our perspective, an environment that facilitates both academic and community-based trialists should be created.

6. Support industry's call for a transparent budgeting process and encourage shared exploration of cost reduction through improved trial operations and recruitment through more relevant and feasible studies. Under pressure from sponsors, our fee schedule has been almost flat since 2004 . The administrative, regulatory, and training burden has grown considerably, without cost recovery, with an estimated $30 \%$ to $50 \%$ increase in work per patient enrolled and fewer patients enrolled per site. With the decline in enrollment and rising costs, many trials are conducted at a financial loss (because fixed costs such as space, staff, and insurance are the same whether 1,10 , or 100 patients are enrolled).

7. Educate the public about clinical trials and raise awareness of the importance of clinical research to patient care. It is through education that we can begin to change the negative perceptions of clinical trials. One example is the US-based Center for Information and Study on Clinical Research Participation (www.ciscrp.org). Trials education is a critical foundation for disease-specific research communication strategies.

8. Increase efforts to harmonize ethics approval and other processes, particularly for multicenter clinical trials. The potential to reduce the cost and administrative burden of multiple reviews while continuing to safeguard human protections is well documented in the literature and by the expert panel reports on how to address this issue. Several countries, including the United Kingdom and Argentina, have invested in clinical research infrastructure. They have funded networks and centralized infrastructure to streamline ethics approval and contracts. This commitment to clinical research focuses on policy and funding of infrastructure. There are many groups working on these challenges including provincially funded efforts to deal with national problems. For example, the cancer network in Ontario has a provincial approval for cancer trials. There are academic research organizations funded by grants that provide research support but at a cost, to sustain their infrastructure. 9. Commission studies to determine effective (and cost-effective) ways to treat rheumatologic diseases. This is especially applicable to public payers. Payers spend billions of dollars on the costs of healthcare but fund very little research. 10. Ensure that new therapies are appropriate for their intended markets; perhaps regulatory authorities should consider whether the results are generalizable to the population in each region.

The CRRC has worked diligently to raise awareness of our challenges and to advocate for change. Of particular importance, its expert protocol reviews have encouraged clinical trials that were pertinent, ethical, and feasible. By the time this editorial is published, the CRRC will have been dissolved. The CRRC is an organization of clinical trial sites and many member sites are closing because of inadequate recruitment in trials (including not accepting trials at sites where they do not reflect practice and/or are not feasible) and financial losses to sites. Because the network is self-sustaining, without arthritis trials it cannot generate revenue. The CRRC leaves an important legacy of templates for GCP in research, budgets, and site-specific feasibility, as well as advocacy to standardize institutional board reviews (ethics reviews) and make them more efficient.

Conducting protocol reviews for every proposed study, the CRRC identified and broadly communicated the challenges with trial designs and hosted several meetings with everyone involved. As seasoned researchers, we have been fortunate to experience breakthroughs in research that have altered the lives of our patients. With the demise of the current paradigm there is an opportunity to explore other trial designs and establish research communities with niche expertise such as in optimizing treatment. The current conduct of pharmaceutical-funded research is not feasible for many research sites and should be changed.

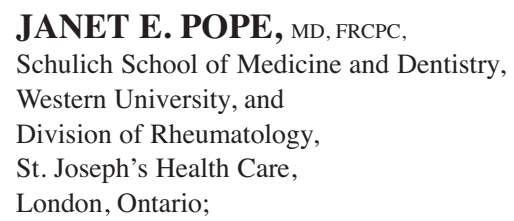

J. CARTER THORNE, MD, FRCPC, Southlake Regional Health Centre, Newmarket, Ontario;

BOULOS P. HARAOUI, MD, FRCPC,

Rheumatic Disease Unit, Institut de Rhumatologie, Montreal, Quebec;

JACOB KARSH, MD, FRCPC,

University of Ottawa, Division of Rheumatology;

EDWARD C. KEYSTONE, MD, FRCPC, Rheumatology Division, Mount Sinai Hospital, University of Toronto;

LINDA BENNETT, BSc,

Executive Director,

Canadian Rheumatology Research Consortium, Toronto, Ontario, Canada.

Address correspondence to Dr. J.E. Pope, 268 Grosvenor St., London, Ontario N6A 4V2, Canada. E-mail: janet.pope@sjhc.london.on.ca The authors have consulted for multiple pharmaceutical companies, including designing trials and reviewing protocols.

Personal non-commercial use only. The Journal of Rheumatology Copyright $\odot$ (2015. All rights reserved. 


\section{ACKNOWLEDGMENT}

The CRRC expresses gratitude to Linda Bennett for her work making clinical trials better for patients.

\section{REFERENCES}

1. Rx\&D, CIHR, ACAHO sponsors. Report from the Canadian clinical trials summit. An action plan to help attract more clinical trials to Canada [Internet. Accessed November 10, 2014.] Available from: www.acaho.org/docs_new/CT\%20Summit/ December\%202012/Action\%20Plan\%20with\%20Appendix/Action Plan_Final.pdf

2. The Standing Senate Committee on Social Affairs, Science and Technology. Canada's clinical trial infrastructure: a prescription for improved access to new medicines. Parliament of Canada. Serial, May 2012. [Internet. Accessed November 10, 2014.] Available from: http://www.parl.gc.ca/Content/SEN/Committee/411/soci/ dpk/01nov12/home-e.htm

3. Karsh J, Keystone EC, Haraoui B, Thorne JC, Pope JE, Bykerk VP, et al. Recommendations for clinical trials of pharmacologic interventions in RA: inclusion criteria and study design. J Rheumatol 2011;38:2095-104.
4. Kay J, Morgacheva O, Messing SP, Kremer JM, Greenberg JD Reed GW, et al. Clinical disease activity and acute phase reactant levels are discordant among patients with active rheumatoid arthritis: acute phase reactant levels contribute separately to predicting outcome at one year. Arthritis Res Ther 2014;16:R40.

5. Bykerk V, Akhavan P, Hazlewood GS, Schieir O, Dooley A, Haraoui B, et al. Canadian Rheumatology Association Recommendations for Pharmacological Management of Rheumatoid Arthritis with Traditional and Biologic Disease-modifying Antirheumatic Drugs. J Rheumatol 2012;39:1559-82

6. Smolen JS, Landewé R, Breedveld FC, Buch M, Burmester G, Dougados $\mathrm{M}$, et al. EULAR recommendations for the management of rheumatoid arthritis with synthetic and biological disease-modifying antirheumatic drugs: 2013 update. Ann Rheum Dis 2014;73:492-509.

J Rheumatol 2015;42:14-7; doi:10.3899/jrheum.140717 